\title{
Development of a Soft X-ray Microprobe for Radiobiology Studies
}

\author{
I.A. Appuhamilage*, D. Adjei, S.S. Alatabi, R. Alnaimi, A. Michette \\ AND S. PFAUNTSCH \\ Department of Physics, King's College London, WC2R 2LS, Strand, London, United Kingdom
}

\begin{abstract}
The King's College London (KCL) first X-ray microprobe (MKI) and the third generation microfocus X-ray sources (MKIII) are intended to be used for various applications including the study of physical and biological interactions at the atomic and molecular scales. The microfocus ultra-soft X-ray sources (MKI and MKIII) with interchangeable targets will provide a superior spatial resolution (a focal spot a few hundreds of nanometres in diameter can be achieved) and the control of the dose delivered to irradiated cells. This will require characterization of the spectra and intensities of the source, measurements of the focus intensities and spot sizes of suitable X-ray optics such as zone plates, grazing incidence microstructured optical arrays and multilayer mirrors.
\end{abstract}

DOI: $10.12693 /$ APhysPolA.125.882

PACS: $41.50 .+\mathrm{h}, 07.85 . \mathrm{Fv}$

\section{Introduction}

Ionizing radiation continuous to play important roles in human society with human population continuously subjected to a range of environmental, occupational and medical exposures [1]. Despite being widely used in cancer radiotherapy applications, ionizing radiation is itself a proven carcinogenesis agent. Understanding how radiation interacts with cells, cellular components and tissues is very important in the studies of radiation-induced cancers. Previously, focused X-rays from continuous-beam microfocus sources have shown that low-dose effects differ significantly from predictions extrapolated from higher doses.

The biological effects of low-energy X-rays are currently studied using, mainly, synchrotron radiation $[2,3]$. However, synchrotron facilities offer limited accessibility and high cost and so many applications will not be widespread, and therefore routinely available as analytical tools, if they are confined to synchrotrons because of the cost of building, maintaining and running of these facilities. They are or have, therefore, become national and increasingly, international facilities and as such are not suitable for the wide-scale development of radiobiology studies. Laboratory X-ray sources can be used to investigate the effects of ionizing radiation on biological cells and tissue to compliment the studies done with synchrotron X-ray sources and heavy-ion systems [2-7]. Since 1990, there has been a renaissance of X-ray microbeams to provide quantitative and mechanistic radiological information that complement the charged particle studies. Currently, there are three microbeam facilities routinely used for biological experiments, which employ X-rays: two are based on laboratory bench X-ray

*corresponding author; e-mail: indika.pathirana@kcl.ac.uk sources (MKII) (Queen's University Belfast and Nagasaki University, Japan) and one has been developed around synchrotron X-ray beams (Photon Factory in Tsukuba, Japan). These previous focused X-rays from continuous-beam microfocus sources have contributed greatly to a recent rapid increase in knowledge of radiobiological pathways and the physical/chemical processes occurring through radiation-induced damage to cells, the subsequent repair and bystander effects. X-ray sources commonly used in radiobiology, among other applications are based on the same principle, with the electrons being directed at a specific target. Scientists have used $\mathrm{X}$-ray tubes with titanium, copper, silver, magnesium, aluminium and carbon targets to generate characteristic $\mathrm{X}$-rays of these materials to irradiate a variety of mammalian cell lines.

Recent development of laboratory microfocus X-ray sources with efficient optics [8] is playing an increasingly important role in understanding of the mechanistic aspects of ionizing radiation effects on DNA double strand breaks $[3,4,6,8]$. The main challenge with this technique is the focusing of the electrons onto a small focal spot on the target to generate X-rays with small focal spot for irradiating part of cellular materials. Limitations in the photon intensity and the available energies from X-ray tube sources prevent definitive characterization of a relationship between photon energy and biological damage. Another major problem with electron impact in X-ray tube sources is the heating of the target material that has major effect on both the X-ray intensity and the system. This limits both the voltage through which the electrons can be accelerated and the number of electrons which can be used, in order to stop the target from melting.

\section{The King's College London contribution}

The main objective of the work at KCL is to re-instate our first microfocus X-ray source (MKI) and to design and develop a third generation microfocus X-ray source (MKIII) which is in progress. The technique for re- 
-instating the MKI is based on the work done by Folkard $[6,9,10]$ and Schettino et al. [11, 12]. The renovation of the MKI will provide ultra-soft X-rays to investigate spatial aspects of the interaction of ionizing with biological samples. This required the modification of the geometry of the Wehnelt cap relative to the cathode position, design of the fine focusing electromagnetic lens for the electron beam, transmission properties of the exit window for the emitted X-rays and monochromatization of X-ray beam by its reflection from the polished silica mirror. The renovated MKI microfocus ultra-soft X-ray source with interchangeable targets will provide a superior spatial resolution (a focal spot a few hundreds of nanometers in diameter can be achieved) and the control of the dose delivered to irradiated cells. This will require characterization of the spectra and intensities of the source, measurements of the focus intensities and spot sizes of suitable X-ray optics, such as zone plates, grazing incidence microstructured optical arrays, and multilayer mirrors.

\subsection{The redesign of the MKI X-ray source}

The King's College London MKI microbeam facility mainly uses electron bombardment X-ray source where electrons are generated by heated thin layer of tungsten filament on a plate of light material and accelerated up to $10-15 \mathrm{keV}$. In the evacuated microfocus tube, the filament current is controlled by means of the Wehnelt (grid) voltages, which is held at a negative potential. The position of the Wehnelt (grid) and its potential play an important role in determining the number of electrons that are emitted by the electron gun. From Fig. 1, it is evident that there is an optimum distance between the filament and the Wehnelt cup in generating optimum amount of electrons.

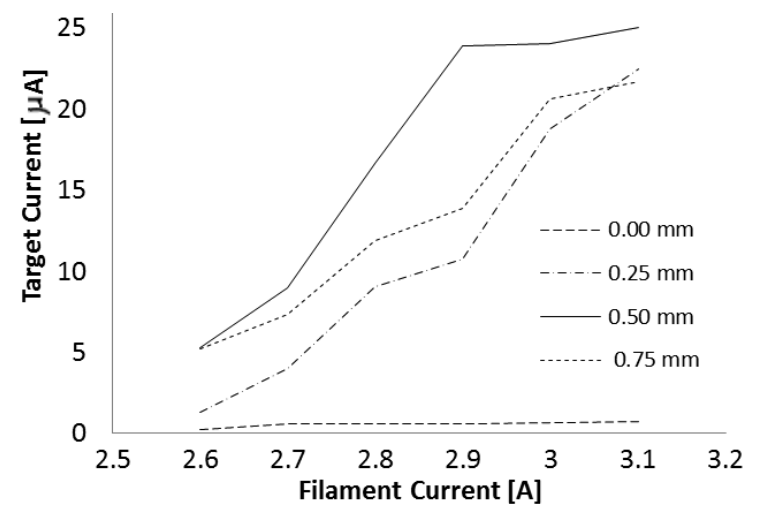

Fig. 1. Effect of filament-Wehnelt cup gap on target current at different filament currents.

\subsection{Focusing electromagnetic lens}

The purpose of this study is to focus electron beam onto a focal spot on the target in order to produce "a point-like" X-ray source for the biological studies. The electron beam as passes through a hole in the anode is directed onto an electromagnetic lens where it is then collimated and focused onto a solid target producing "a point-like" X-ray source. The renovated MKI electromagnetic lens consists of 180 turns of copper wire coiled around a soft iron with and a variable current up to $2 \mathrm{~A}$ to increase electromagnetic field strength for the focusing of the electron beam. The electron beam focal spot size is simulated using Field Precision (Field Precision, NM, USA). The design of the single pole magnetic lens was drawn in Mesh drawing editor of the Field Precision which constructs logical structures and analysis of the boundary vectors of the lens defined. The PerMag code also employs the finite-element methods to calculate the magnetostatic fields in the coil geometry.

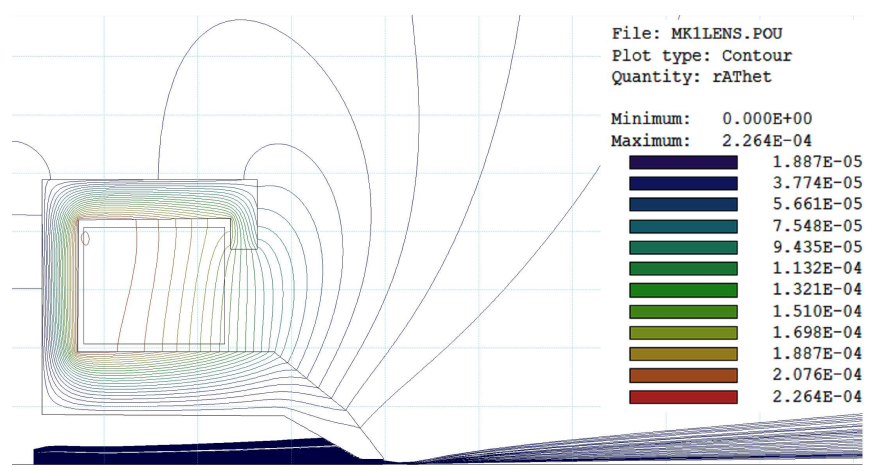

Fig. 2. Electromagnetic lens field strength to focus electron beam onto the target.

Figure 2 shows the electron beam focusing using single pole lens from the simulation done by the Field Precision Software. The estimated maximum magnetic field strength was $0.1 \mathrm{~T}$ to demagnify the electron beam to $\approx 20 \mu \mathrm{m}$ diameter.

2.3. Monochromatization of the X-ray beam

The electron beam focused onto the carbon target produces both characteristic $\mathrm{X}$-rays and continuous bremsstrahlung. A single-sided polished silicon mirror is

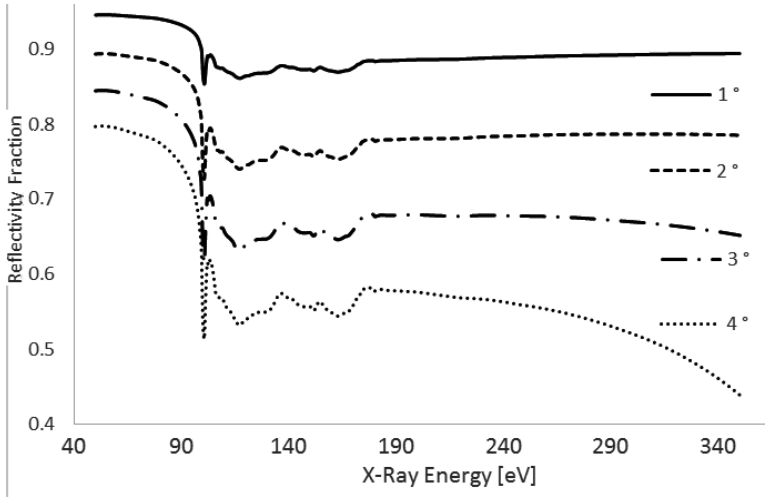

Fig. 3. Reflectivity fraction of a silica mirror for incident X-ray energies at different grazing angles.

used as a monochromator to select carbon-K line from the spectrum emitted from the graphite target when bombarded with electrons with energies of $10-15 \mathrm{keV}$. Using the phenomenon of total internal/external reflection, 
which occurs at small incident angles and low energies, it is possible to use a mirror to "filter" the X-ray spectrum. The ability of the silicon mirror to eliminate high energy $\mathrm{X}$-rays while maintaining high reflectivity for carbon- $K$ alpha radiation is determined with the graphite target under bombardment for $10 \mathrm{kV} / 300 \mu \mathrm{A}$ electron beam with the specific positions of the silica mirror inside the $\mathrm{X}$-ray chamber. A simulation of the reflectivity of a silica mirror for incident X-ray energies at different grazing angles is shown in Fig. 3.

\subsection{The X-ray microprobe exit window}

The $\mathrm{X}$-ray exit window is a $100 \mathrm{~nm}$ thick silicon nitride window with an area of $1 \times 1 \mathrm{~mm}^{2}$. The window is strong enough to hold an atmosphere of high pressure difference and present a high transmission for low-energy photons. A simulation of the transmission fraction for the different vacuum windows available for the work were performed to select a material appropriate for the X-ray microprobe system using CASINO Monte Carlo software [13].

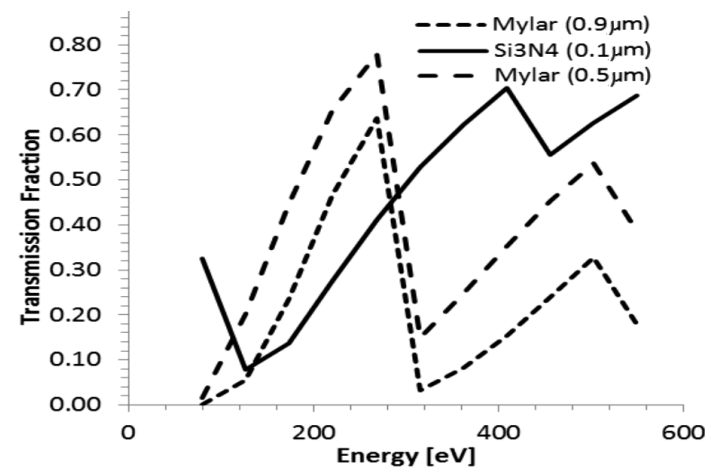

Fig. 4. Low energy X-ray transmission fraction for different vacuum windows.

It is shown in Fig. 4 that a mylar $\left(\mathrm{C}_{10} \mathrm{H}_{8} \mathrm{O}_{4}\right)$ with $(0.5 \mathrm{~mm})$ shows the highest transmission fraction for the carbon- $K$ target energy. However, due to the high vacuum maintained in the chamber, it was prudent to use $0.1 \mu \mathrm{m}$ silicon nitride $\left(\mathrm{Si}_{3} \mathrm{~N}_{4}\right)$ giving a transmission of $41-42 \%$ for the carbon- $K$ X-ray.

\subsection{Development of epi-flourescence microscope}

The imaging system is mounted above the microfocus source on the optical bench. It comprises of a custom-built epi-illuminated polarized microscope with an encoded, rotary objective lens turret and a ProScan motorized $x-y$ transmission stage for closed-loop sample positioning with sub- $\mu \mathrm{m}$ precision.

\subsection{The MKI microprobe optics}

The MKI is designed for the production of micrometer-sized X-ray spots capable of irradiating cellular and significant subcellular targets of biological samples. The diffraction technique of focusing X-ray beams have so far been favoured for microbeam applications mainly due to their easily application. Using suitable X-ray optics such as zone-plates, grazing incidence micro-structured optical arrays and multilayer mirrors, the microfocus X-ray source (MKI) will be capable of delivering a small $5 \mu \mathrm{m}$ spot of X-rays. Figure 5 shows a laboratory setup of the MKI facility for biological studies.

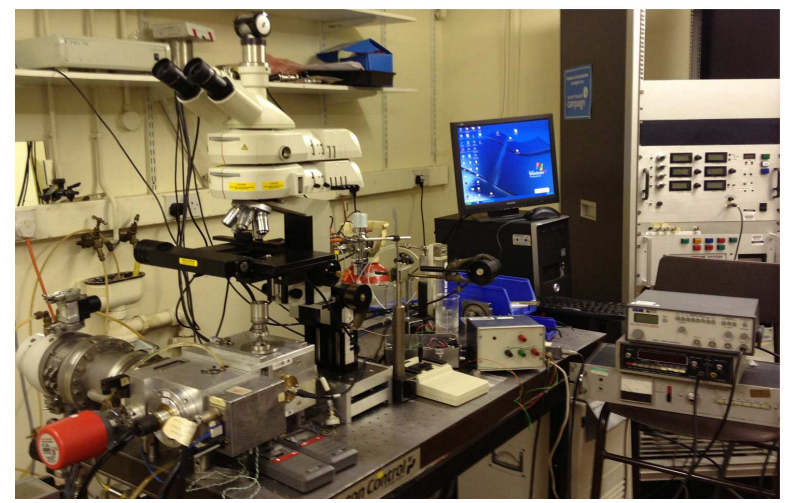

Fig. 5. MKI laboratory setup.

\subsection{The ultra-soft X-ray detector for the MKI}

A custom-made proportional counter has been developed to detect carbon- $K$ X-rays. This counter has features just as any proportional counter, consisting of a thin conducting wire held at positive voltage surrounded by a grounded metal cylinder, except that our proportional counter includes a gas-filled chamber fitted with a $0.5 \mu \mathrm{m}$ thick mylar film placed at the chamber window. Two small, $1 \mathrm{~mm}$ gauge tubes pass through holes in the cathode wall and allow the chamber to be continuously flushed with P10 gas; a mixture of $10 \%$ methane in argon. X-rays penetrate the window and pass into the gas inside where interactions with the gas atoms result in the creation of a number of ion pairs (electrons and partially ionized gas atoms). The detection of each photon (an event) results in a discrete signal in the associated electronics. The novel counter is found to operate with a linear response at $8-9 \mathrm{~mL} \mathrm{~s}^{-1}$ of the $\mathrm{P} 10$ gas flow rate at $1900 \mathrm{~V}$.

\subsection{The King's College London MKIII}

The King's College MKIII is the third generation of X-ray source, intended to offer design and performance over the second (MKII) and first (MKI) generation sources for various applications including the study of physical and biological interactions at the atomic and molecular scales. This will require electron beam spot-size at target $<1 \mu \mathrm{m}$ for continuous high-brightness $\mathrm{X}$-ray sources, since the spectral brightness is inversely proportional to the source size, which is typically only a few $\mu \mathrm{m}$ larger than the electron beam focus. The design of the MKIII is also to achieve a high beam current $(1 \mathrm{~mA})$ to produce high $\mathrm{X}$-ray intensities with larger range of beam voltage $(1-30 \mathrm{kV})$ with suitable cooling systems more than that of the MKII and MKI.

The innovative aspect of MKIII is the target configuration which allows the translation of the targets to move in $x, y, z$-directions using a motorized system. The MKIII will allow a considerable smaller focal sizes, $\approx 1 \mu \mathrm{m}$, 
increasing the range of applicability of the source with micro-structured optical array configuration. The design, construction and characterization of the MKIII microfocus probe is in progress.

\section{Conclusion}

It is clear that the development of microprobes using table-top X-ray sources are playing important role in the studies of physical and biological interactions at atomic and molecular scales. The aim of the work presented here is to illustrate the methodology of utilizing these sources in such applications. It is obvious that laboratory X-ray sources can well be used to study the ionized radiation effect on biological cells and tissue just as successful as synchrotron X-ray sources and heavy-ion systems. Investigation results on the effect of $\mathrm{X}$-ray radiation on the nucleus of biological and human cells using the source development described in this work will be published in our next paper which cover different type of targets as well as a variety of cells such as HeLa cells and Chinese Hamster V79 cells. The complete development of the King's College MKI and MKIII with a novel target and considerably smaller X-ray focal spot sizes will increase the range of applicability of the sources which in turn will allow the study of radiation effect on these cells and cellular components of biological systems.

\section{Acknowledgments}

The authors gratefully acknowledge funding from the EXTATIC - Erasmus Mundus Joint Doctoral Programme (D.A.), CARA - the Council for Assisting Refugee Academics - (S.A.) and Centrala studiestödsnämnden CSN Sweden (R.A.).

\section{References}

[1] ICRP, Radiological Protection in Biomedical Research, ICRP Publication 62, Pergamon Press, Oxford 1992.
[2] Y. Kobayashi, T. Funayama, N. Hamada, T. Sakashita, T. Konishi, H. Imaseki, K. Yasuda, M. Hatashita, K. Takagi, S. Hatori, K. Suzuki, M. Yamauchi, S. Yamashita, M. Tomita, M. Maeda, K. Kobayashi, N. Usami, L. Wu, J. Radiat. Res. 50, A29 (2009).

[3] A.C. Thompson, E.A. Blakely, K.A. Bjorstad, P.Y. Chang, C.J. Rosen, D. Sudar, R.I. Schearz, Radiat. Res. 161, 101 (2004).

[4] N. Hamada, G. Schettino, G. Kashino, M. Vaid, K. Suzuki, S. Kodama, B. Vojnovic, M. Folkard, M. Watanabe, B.D. Michael, Radiat. Res. 166, 31 (2006).

[5] G. Schettino, M. Folkard, B.D. Michael, K.M. Prise, Radiat. Res. 163, 332 (2005).

[6] M. Folkard, G. Schettino, B. Vojnovic, S. Gilchrist, A.G. Michette, S.J. Pfauntsch, K.M. Prise, B.D. Michael, Radiat. Res. 156, 796 (2001).

[7] F. Bortolotto, D. Batani, F. Previdi, L. Rebonato, E. Turcu, R. Allott, Eur. Phys. J. D 11, 309 (2000).

[8] A.G. Michette, P.D. Prewett, A.K. Powell, S.J. Pfauntsch, K.D. Atkinson, B. Boonliang, J. Phys. IV 104, 277 (2003).

[9] M. Folkard, B. Vojnovic, G. Schettino, K. Atkinson, K.M. Prise, B.D. Michael, Variable-Energy Soft X-ray Microprobe to Investigate Mechanisms of Radiation-Induced Bystander Effects, DOE Low Dose Radiation Program Workshop IV, 2003.

[10] M. Folkard, B. Vojnovic, K.M. Prise, K. Atkinson, G. Schettino, A.G. Michette, S. Gilchrist, R. Sunderland, B.D. Michael, Radiat. Res. 161, 88 (2004).

[11] G. Schettino, M. Folkard, K.M. Prise, B. Vojnovic, B.D. Michael, Radiat. Prot. Dosim. 99, 287 (2002).

[12] G. Schettino, M. Folkard, K.M. Prise, B. Vojnovic, K.D. Held, B.D. Michael, Radiat. Res. 160, 505 (2003).

[13] B.L. Henke, E.M. Gullikson, J.C. Davis, At. Data Nucl. Data Tables 54, 181 (1993). 